REVISTA DE LITERATURA E CULTURA RUSSA

\title{
Contre le bourgeois! Levinas lecteur de Dostoïevski et Tolstoï
}

\section{Against the bourgeois! Levinas reader of \\ Dostoevsky and Tolstoy}

Autor: Yoann Colin

Edição: RUS Vol. 12. № 18

Data: Abril de 2021 


\section{Contre le bourgeois! Levinas lecteur de Dostoïevski et Tolstoï}

Yoann Colin*

Résumé: Cet article vise à montrer la convergence entre la façon dont Dostoïevski et Tolstoï, qui furent des écrivains incontournables dans la formation de la pensée de Levinas, esquissent une figure du bourgeois et du désembourgeoisement qui consonne avec celle qu'élabore ce dernier, en particulier au début de son œuvre.

\begin{abstract}
This article aims to show the convergence between the way Dostoevsky and Tolstoy, who were indispensable writers in the formation of Levinas's thought, sketch a figure of the bourgeois and the de-bourgeoisness that consones with that which he develops, especially at the beginning of his work
\end{abstract}

Mots-clés: Bourgeois; Levinas; Dostoïevski; Tolstoï; Russe Keywords: Bourgeois; Levinas; Dostoevsky; Tolstoy; Russian 
* Yoann Colin a poursuivi sa formation en classe préparatoire au lycée Fénelon à Paris et a obtenu une équivalence de licence de lettres modernes et licence de philosophie. II a ensuite obtenu une maitrise et un master 2 en philosophie à la Sorbonne. II enseigne la philosophie au lycée depuis 2007. II a obtenu en 2011 une licence de théologie catholique à Strasbourg, où il a soutenu sa thèse de philosophie en 2020. E-mail: yoanncolin_538@hotmail. com evinas revendique un héritage de la culture russe, ${ }^{1}$ principalement littéraire. Il s'agit ici de voir en quoi la littérature russe antibourgeoise, a pu influencer Levinas, en s'appuyant sur la dénonciation des bourgeois dans les grandes œuvres de cette littérature, qu'a méditées Levinas. Levinas reconnait qu'il a appris à se poser les questions importantes qui le menèrent à la philosophie dans les grands romans russes. ${ }^{2}$ Comme le dit R. Sparadro, «le lien qui unit Levinas aux personnages des romans russes est la certitude que le problème éthique est l'événement fondamental de l'expérience humaine, autrement dit "l'éthique comme philosophie première" ». ${ }^{3}$ Cet article de R. Sparadro évoque la présence d'un certain nombre de textes russes sous la plume de Levinas et défend la thèse que la «littérature russe fait partie de la formation culturelle de Levinas». ${ }^{4}$ Il nous semble que ce sont surtout Tolstoï et Dostoïevski qui l'ont influencé. Et, plus particulièrement, nous défendons la thèse que la conception lévinassienne du bourgeois est étroitement tributaire de la représentation de cette figure chez Tolstoï et Dostoïevski.

Le personnage du bourgeois est présent dans la littérature russe, bien que la structure de la société russe diffère fortement de celle des pays situés à l'ouest de l'Europe. La Russie

\footnotetext{
1 Abensour souligne que Tolstoï et Dostoïevski ont été particulièrement importants pour Levinas, y compris aussi sur le plan politique (Abensour, 2011, p. 24). Voir également Marie-Anne Lescourret, Emmanuel Levinas, Paris, Flammarion, 1999, pp. 42-48.

2 Voir en particulier ses entretiens de Levinas avec F. Poirié. II répond à la question «qu'est-ce qui vous a mené à la philosophie?» en disant: «Je pense que ce sont tout d'abord mes lectures russes. C'est précisément Pouchkine, Lermontov et Dostoïevski, surtout Dostoïevski. Le roman russe, le roman de Dostoïevski et de Tolstoï, me paraissait très préoccupé des choses fondamentales. Livres traversés par l'inquiétude, par l'essentiel, l'inquiétude religieuse, mais lisible comme quête du sens de la vie. [...] L'amour-sentiment des livres, c'est certainement là mes premières tentations philosophiques». (Poirié, 1987, p. 69).
}

3 Sparadro, 2012, p. 43.

4 lbid.. 
du XIXe siècle n'est un pays ni libéral, ni capitaliste, sauf dans le cœur de ceux qui prônent un modèle européen. Le monarque a un pouvoir absolu, qu'il n'a plus en occident au XIXe siècle. Le Tsar représente Dieu, pas le peuple. L'ordre social est constitué par la «Table des rangs» de 1722 qui ne peut pas être directement traduite en "classes» par lesquelles on étudie la structure des sociétés occidentales modernes. D'où la difficulté de cerner le bourgeois par sa position sociale et d'en définir le contour sociologique. Reste que des figures bourgeoises abondent dans la littérature russe, en particulier parmi les intendants des nobles de province qui s'efforcent d'augmenter, sans mesurer toujours les moyens employés, les revenus d'un domaine. Un tel personnage est un topos de la littérature russe du XIXe siècle, qu'on peut assimiler à une figure de bourgeois, en ce qu'il laisse de côté la morale et tout ce que n'est pas profit financier et économique. Les écrivains russes auront ainsi leur bourgeois à caricaturer, chercheurs de profits négligeant tout ce qui n'est pas l'objet de leurs «affaires». Nous nous proposons de déterminer la figure du bourgeois dans certains écrits de Tolstoï et Dostoïevski et de rapprocher cette figure de celle qui habite la pensée lévinassienne. Pour ce faire, nous mettons en résonance trois formes de la présence du bourgeois chez Levinas et leur proximité avec des passages des œuvres de ces derniers.

\section{Le portrait du bourgeois: similitudes entre les bourgeois de l'Idiot et le début de De l'évasion}

Dans les textes de Dostoïevski, le terme de bourgeois n'est pas toujours univoque. Il peut désigner, dans un sens quasiment objectif, un homme ou un personnage appartenant à la classe sociale bourgeoise ${ }^{5}$ ou des personnages animés d'un

5 Ainsi le personnage de Parfione Rogojine, fils de Sémione Rogojine, «bourgeois honoraire héréditaire, qui est mort (...) en laissant une fortune de deux millions et demi à ses héritiers» (Dostoïevski, 1994, p. 9). 
esprit bourgeois. Pour ne s'en tenir qu'à L'Idiot, on peut voir de relativement grands bourgeois, liés avec la noblesse (les Epantchine), des petits-bourgeois (comme Varvara Ardalionovna Ptisyna, son époux Ptisyne et son frère Gavrila Ardalionovitch), et le fils d'un riche marchand (Rogojine). Plus que leur appartenance à une classe particulière et le mode de vie ou le rapport au travail qu'ils peuvent avoir, ce qui les caractérise comme bourgeois, c'est leur état d'esprit. Ainsi le général Epantchine, qui s'est enrichi dans les affaires et n'accorde d'importance qu'à elles, est décrit comme un propriétaire, riche qui a de hautes relations et qui pense «toujours à l'avenir». ${ }^{6} \mathrm{Il}$ se sert de ses affaires comme prétexte quand cela l'arrange. ${ }^{7}$ Rogojine, fou de Nastasia Philippovna, veut l'acheter, ${ }^{8}$ et sans cette dévorante et violente passion, férocement égoïste, il serait un vulgaire bourgeois banal. ${ }^{9}$ De leur côté, les petits-bourgeois ne cherchent qu'à réussir, parvenir, se hisser au sommet de la pyramide sociale, rêvant et économisant, n'aspirant qu'à sortir de leur (médiocre) ordinaire. Ainsi Ptysine, usurier se défend contre l'accusation portée par son beau-frère en essayant de le convaincre «qu'il ne faisait rien que d'honnête (...), que l'argent était à ce taux-là, il n'y était pour rien ; que sa façon de procéder était honnête et probe»:10 en bourgeois, il tient à avoir sa conscience pour lui ; et, en bourgeois également, il a ses rêves - qui sont tout autant rêve que calcul - de réussite sociale. ${ }^{11}$

6 Ibid., p. 18

7 Ibid., p. 61.

8 Ibid., p. 195.

9 C'est du moins ce que pense le prince Mychkine qui lui confie: «l'idée m'est venue que, si cette passion ne te torturait pas, tu serais devenu, et en fort peu de temps, pareil à ton père. Tu te serais renfermé dans cette maison avec une femme obéissante et muette ; tu n'aurais fait entendre que de rares et sévères propos ; tu n'aurais cru à personne et n'aurais pas même éprouvé le besoin de te confier ; tu te serais contenté d'amasser de l'argent dans l'ombre et le silence» (ibid., p. 260). Notons également que le personnage de Totski est également un homme riche, profondément égoïste, qui veut satisfaire son désir pour les femmes, sans aucun souci d'autrui (ibid., p. 46-47).

10 Ibid., p. 565.

11 «"je ne deviendrai pas Rothschild, ajoutait-il en souriant, et n'ai pas de motif de le devenir ; j'aurai une maison, peut-être même deux, sur la Liteïnaïa, et je m'en tiendrai là". II pensait à part soi: "Qui sait ? peut-être bien trois aussi!" mais il n'exprimait jamais ce rêve et le gardait 
Esquissées à grands traits, ces caractéristiques du bourgeois sont proches de celles que Levinas lui attribue au début d'une œuvre de jeunesse, De l'évasion. En effet, dans ce texte, ce qui caractérise le bourgeois, c'est d'une part l'obsession des "affaires», Levinas écrit que le bourgeois "se soucie d'affaires et de sciences comme d'une défense contre les choses et l'imprévisible qu'elles recèlent». ${ }^{12}$ Cette attitude du bourgeois est développée et expliquée à la phrase suivante: «contre l'avenir qui introduit des inconnues dans les problèmes résolus sur lesquels il vit, il demande des garanties au présent» ${ }^{13}$ Le bourgeois agit dans le présent de façon à le dominer. L'inquiétude et le souci ne portent pas sur le présent mais sur l'avenir, pour contrer le potentiel effet délétère du futur. En faisant en sorte que son avenir soit aussi certainement connu et, par l'assurance, aussi confortable et sécurisé que son passé, le bourgeois vit une existence quasiment linéaire, où rien d'extérieur ne vient troubler la maîtrise qu'il exerce sur le monde.

Ces personnages romanesques font voir que leur seule préoccupation est centrée sur leur être, sur la satisfaction de leurs désirs, quitte à dominer l'autre plutôt que de chercher son amour, semblable en cela au bourgeois lévinassien. Le conflit dont la philosophie occidentale moderne, «bourgeoise» même aux yeux de Levinas, est issue, comme l'explique Levinas dès le début de De l'évasion, oppose l'homme au monde et non l'homme à lui-même. Levinas remarque qu'elle se définit par un idéal de paix avec soi-même, sans tenir compte du sort des autres, l'amour authentique y étant interdit, puisque ce que recherche le sujet de cette philosophie, c'est le triomphe du héros, qui «lutte», ${ }_{1}^{14}$ qui vainc plus qu'il n'aime, qui possède et s'approprie plus qu'il ne s'abandonne. Levinas présente le romantisme comme ne s'écartant pas de cet idéal de paix, celle du moi unifié, qui se repose sur lui-même, autrement dit du sujet chez lui, «installé», qui ne se laisse pas déranger, par le

dans son for intérieur» (ibid., p. 565).

12 Levinas, 1998a, p. 92.

$13 \mathrm{lbid}$.

14 lbid. 
monde et les autres, le sujet autonome et tributaire de rien ni personne. La revendication de l'autonomie par la philosophie traditionnelle est opposée à l'hétéronomie (dont l'amour serait un cas de figure), au fait de se laisser toucher et influencer par l'extérieur et les autres.

Les portraits de bourgeois de L'idiot, témoignent du souci de pouvoir revendiquer une bonne conscience, dépourvue de remords ou de culpabilité. De la même façon, dans son portrait du bourgeois qui ouvre De l'évasion, Levinas écrit que, pour justifier son action sur le monde, le bourgeois s'appuie sur sa bonne conscience manifeste, sur le fait que ses actions ne paraissent pas rencontrer d'obstacle en lui, qu'il semble en accord avec lui-même quand il décide. Autrement dit, le bourgeois ne fait pas d'effort pour se changer lui-même, car il prétend être déjà comme il devrait être et n'a donc pas besoin de se réconcilier avec lui-même puisqu'il est déjà unifié et réconcilié: «son manque de scrupule est la forme honteuse de sa tranquillité de conscience». ${ }^{15}$ Le bourgeois est ainsi enfoncé dans l'économie, soustraite à la morale, fixée sur l'enrichissement. La bonne conscience ou la tranquillité de conscience du bourgeois est une carapace pour faire ce qui est conforme à sa volonté de posséder, de maitriser. Levinas explique que si les bourgeois ont bonne conscience, ce n'est pas parce que leur conscience est innocente ou irréprochable, mais parce qu'ils ne l'entendent pas ou l'ont déformée. Comme les bourgeois n'ont pas mauvaise conscience, alors qu'ils le devraient, pour Levinas, c'est le défaut de mauvaise conscience qui est pris pour une bonne conscience. Ce n'est pas parce qu'il n'a pas de scrupule que le bourgeois a bonne conscience, mais c'est parce qu'il ne la prend pas en compte. Il est celui qui arrive à justifier son attitude par des raisons, tout en étant traversé par un sentiment, auquel il ne prête aucune attention, de culpabilité parce qu'il ne fait pas droit à la responsabilité pour l'autre qui lui incombe. 


\section{L'essai sur le bourgeois: du portrait du bourgeois à la dénonciation de la philosophie occidentale moderne bourgeoise}

Mais, le plus souvent, et de façon plus catégorielle, le bourgeois est, sous la plume de Dostoïevski, la figure de l'homme occidentale moderne. Il incarne l'esprit occidental, notamment dans certains textes théoriques. Dans le feuilleton Remarques d'hiver sur des impressions d'été, publié en 1863, Dostoïevski écrit un «Essai sur le bourgeois» dans lequel la caractérisation du bourgeois est avant tout spirituelle: il est celui qui a l'esprit bourgeois, et non celui appartiendrait à une classe sociale déterminée. Et Dostoïevski qualifie l'action essentielle du bourgeois, à l'époque où il le décrit, comme celle de «se ratatiner», ${ }^{16}$ ce qu'on peut expliciter par la volonté d'un repli sur soi, sur ses intérêts privés, égoïstes et principalement financiers. ${ }^{17} \mathrm{Ce}$ qui en dit long sur l'importance de l'argent et le souci de la seule existence personnelle - et, corrélativement, sur l'indifférence à autrui ou à la collectivité. De plus, comme le note Dostoïevski, «ce qu'il faut, c'est que tout resplendisse de vertu» ${ }^{18}$ Est ainsi soulignée, avec véhémence, l'importance de l'apparence. Le bourgeois n'est pas le vertueux, mais celui qui se fait passer pour tel. Et à ce culte ostensible de la vertu s'ajoute l'appétit immodéré, et canonique dans les descriptions du bourgeois, pour l'enrichissement. ${ }^{19}$ Ainsi Dostoïevski brosset-il le portrait du bourgeois, en envisageant son obsession de

16 Dostoïevski, 1969, p. 1470.

17 Ce que confirment un grand nombre de remarques qui vont dans ce sens. Voir par exemple: «Voilà, aujourd'hui je fais encore un petit peu de bénéfice dans ma boutique, si Dieu veut j'en ferai encore un peu demain, peut-être aussi après-demain, la grâce du Seigneur aidant... Et puis après, après, pourvu que j'arrive au plus vite à mettre au moins un morceau de côté - après moi le déluge» (ibid., p1471)

18 lbid.

19 Ibid., p. 1473. 
la réussite financière, dissimulée par le masque de la vertu. Ce masque vantant devoir, moralité et justice couvre en réalité des méfaits et une totale indifférence au dénuement de l'autre. ${ }^{20}$

À partir de l'analyse de l'ordre bourgeois de la société qui sert les intérêts propres de la bourgeoisie sous couvert de servir l'intérêt de tous, Dostoïevski se livre à un commentaire décapant de la devise républicaine, en montrant, en des termes proches de ceux du Marx de La Question juive que la liberté revendiquée par la démocratie bourgeoise est non seulement vaine car abstraite, mais que sous cette proclamation de la liberté pour tous, il y a, en réalité, asservissement pour la majorité des hommes, ceux qui ne possèdent pas assez pour «faire fonctionner» leur liberté. ${ }^{21}$ Ainsi, ce n'est plus seulement le portrait du bourgeois que peint Dostoïevski, mais sa conception du monde, telle qu'elle s'illustre dans ce qu'il croit être la philosophie occidentale moderne, individualiste. Paradoxalement le bourgeois, individualiste, ne peut donner la pleine mesure de son être qu'au sein du monde qu'il habite - ou, pour mieux dire, qu'il exploite, en dissimulant du mieux qu'il peut cette exploitation. Dans la suite de son analyse, Dostoïevski critique la conception occidentale de l'égalité, non seulement sur le plan politique, mais même ontologique ou métaphysique. Pour lui, le tempérament occidental en général ne comporte pas l'égalité.

Il comporte le principe personnel, le principe du particulier, le principe d'un constant effort d'autoconservation, d'auto-acquisition, d'autodétermination dans son propre moi, d'opposition de ce moi à toute nature et à tous les autres hommes, en tant qu'être individuel affirmant son propre droit et qui se veut totalement égal et de valeur égale à tout ce qui n'est pas lui. Alors, ce n'est pas de cette individualisation que pouvait naître de la fraternité. Pourquoi ? Parce que dans la fraternité, dans la vraie fraternité, ce n'est pas la personnalité individuelle, ce n'est pas le moi qui doit se

20 Ibid., p. 1475.

21 «Qu'est-ce que la liberté ? Le droit égal pour tous de faire tout ce qu'ils veulent dans les limites de la loi. Quand peut-on faire tout ce qu'on veut ? Quand on a un million. La liberté donne-t-elle à chacun un million? Non. Qu'est-ce qu'un homme sans un million? Un homme sans un million n'est pas celui qui fait tout ce qu'il veut, mais celui duquel on fait tout ce qu'on veut» (ibid., p. 1476). 
soucier de son droit d'avoir autant de valeur et de poids que tout le reste, c'est au contraire tout ce qui reste qui devrait de lui-même venir à cette personnalité exigeant ses droits, à ce moi individuel, et qui devrait de lui-même, sans en être prié, le reconnaître égal en valeur et en droits à tout ce qu'il y a au monde en dehors de lui. Et ce n'est pas tout: elle-même, cette individualité exigeante et révoltée, devrait avant tout se sacrifier elle-même, sacrifier tout son moi à la société, et non seulement ne pas réclamer son droit, mais au contraire le livrer sans condition à la société. Mais l'individu occidental n'est pas fait à pareil ordre de choses: il exige de haute lutte, il revendique son droit, il veut partager - et cela ne fait pas de la fraternité. ${ }^{22}$

La critique de Dostoïevski semble ainsi préluder à la critique lévinassienne du cœur de la philosophie occidentale. La philosophie occidentale est ici pour Dostoïevski l'autre d'une philosophie, personnaliste en un certain sens, à laquelle il aspire. $A$ contrario de cette mise en avant du moi occidental et individualiste, prêt à tout pour obtenir ce à quoi il croit avoir droit, Dostoïevski développe ce vers quoi devrait tendre l'homme, pour manifester, de la meilleure façon qui soit, son humanité et sa grandeur. Il faut, d'après lui, être capable de se sacrifier sans en être prié pour tous, c'est le «signe du plus haut développement de la personnalité, de sa plus haute puissance, de sa plus haute maitrise de soi, de sa plus haute capacité de libre arbitre». ${ }^{23}$ Il définit cette forme d'humanité, de façon paradoxale, comme «la loi de la nature, c'est vers quoi l'homme est naturellement tiré». ${ }^{24}$ Toute proportion gardée, cette «loi» est l'analogue de l'aspiration à la sainteté comme modèle de l'éthique pour Levinas (qui, lui, cependant ne voit rien de «naturel» dans le mouvement contraire à la pente bourgeoise et individualiste de l'occident qui cherche à posséder et maîtriser). Le problème, dont on retrouve là encore des échos dans la réflexion de Levinas, c'est quand on nourrit «la moindre arrière-pensée d'avantage personnel». ${ }^{25}$ Ainsi, «si par exemple

22 Ibid., p. 1477.

23 Ibid., p. 1478

$24 \mathrm{lbid}$.

$25 \mathrm{lbid}$. 
je m'offre tout entier en sacrifice pour tous, alors il faut que je me sacrifie entièrement, pour de bon, sans esprit de profit, et non pas que je me dise que puisque je me sacrifie tout entier à la société, la société à son tour doit se donner tout entière à moi», ${ }^{26}$ ce qui est impossible si on y pense consciemment. Aussi la fraternité ne doit pas être une construction, mais une donnée de nature, qui doit rester inconsciente, à l'insu. Aussi Dostoïevski en conclut-il que la «fraternité ne se fabrique pas, il faut qu'elle se fasse d'elle-même, qu'elle soit dans le naturel, qu'elle réside inconsciente dans le tempérament de toute la race, en un mot qu'l y ait un principe d'amour fraternel: il faut aimer». ${ }^{27}$ Ce qui signe l'échec prévisible du socialisme occidental qui entend construire cette fausse fraternité.

En effet, dans De l'évasion, Levinas analyse la philosophie traditionnelle, qu'il qualifie de «bourgeoise», ou du moins de solidaire de «l'esprit bourgeois», l'individu y est appelé à desserrer l'étreinte de la réalité étrangère qui l'étouffe pour assurer le plein épanouissement de sa réalité propre. La vocation humaine du sujet de la philosophie occidentale est purement individuelle, voire individualiste ; en tous cas, elle place le sujet et l'individu au centre (il doit atteindre une forme d'accomplissement personnel propre (sa «réalité propre», qui s'oppose à la «réalité étrangère» qu'il doit tenir à l'écart et dont il doit s'extirper ${ }^{28}$ ). L'homme, dans la philosophie traditionnelle et bourgeoise, semble voué à ne pas agir sur lui-même, mais sur le monde qu'il tente de maîtriser voire d'asservir.

Levinas note également que "cette conception du moi comme se suffisant à soi est l'une des marques essentielles de l'esprit bourgeois et de sa philosophie». ${ }^{29}$ On voit ici le mouvement par lequel Levinas dénonce la fausse route que prend la philosophie traditionnelle, qui, s'attache à ce sujet égoïste, autarcique et autonome qu'est le moi, considéré comme bourgeois par Levinas. Contre cette philosophie occidentale mo-

26 Levinas, 1998a, p. 91.

27 Ibid.

$28 \mathrm{Ibid}$.

29 Ibid., p. 91-92. 
derne, considérée comme bourgeoise, Levinas assigne à une autre philosophie le but de déranger le confort du bourgeois qui ne s'intéresse qu'à lui et à son bien-être personnel. Se laisser déranger serait, pour la raison, accepter que soient ébranlées ses catégories et de se soucier effectivement du sort de l'autre. La philosophie que recommande Levinas, et l'usage de la raison qu'elle fait, est nommée ici «vigilance». La vigilance serait la façon non bourgeoise, désembourgeoisée, parce que détachée du Même et de l'identité du sujet:

La vigilance - réveil dans l'éveil - signifie la dé-fection de l'identité, ce qui n'est pas son extinction mais sa substitution au prochain - ordre ou désordre de la raison n'est plus ni connaissance ni action mais où désarçonnée par Autrui de son état - désarçonnée du Même et de l'être - elle est en relation éthique avec autrui, proximité du prochain. ${ }^{30}$

Et comme pour Dostoïevski cette autre philosophie tendrait à faire de l'amour de l'autre la vocation d'une philosophie devenue profondément éthique.

\section{D'une captivité à l'autre: le prisonnier est plus libre que le bourgeois}

Chez Tolstoï aussi de nombreux textes fustigent ou déplorent l'existence ou l'état d'esprit des bourgeois entendus comme petits-bourgeois. Il nous paraît pertinent de mettre en rapport le récit «Captivité», qu'écrit Levinas sur sa détention en Allemagne, avec la description de la quasi-conversion de Pierre Bézoukof en prison dans Guerre et paix. Ce dernier, enrichi tout d'un coup, se trouve en quête du sens de la vie, en vivant dans l'opulence et la haute société. Cherchant ce qui pourrait répondre à son questionnement existentiel, il éprouve un sentiment de honte quand il se rend compte qu'il a fait le bien, mais qu'il aurait pu en faire encore plus. Riche, il peut soulager la misère de ses serfs dont il se sent responsable, mais est bientôt gagné par le sentiment de ne pas en avoir

30 Levinas, 1998b, p. 60. 
fait assez pour eux. ${ }^{31}$ Pierre se rend bien compte qu'il n'en a et n'en aura jamais fait assez, d'où, malgré sa richesse, le sentiment d'inanité qui caractérise son existence tant qu'il n'a pas encore trouvé le sens de la vie. D'où sa fuite dans le divertissement, en particulier dans l'alcool. Pendant l'occupation de Moscou par l'armée française, Pierre passe devant des gens dont l'habitation est en flamme. Une mère implore qu'on aille chercher son enfant resté à l'intérieur. Pierre réussit à sortir l'enfant de la maison en flamme. Voulant ensuite protéger des civils malmenés par des soldats, il s'interpose et est arrêté. Capturé, Pierre assiste à une exécution de prisonniers, ce qui engendre en lui la perte de confiance en l'homme et en la vie. En captivité, Pierre trouve le sens de la vie, ${ }^{32}$ après l'avoir cherché, grâce à Karataïev. Il supporte sa situation avec joie, malgré les privations. Cela conduit à changer son regard sur le monde, à voir l'essentiel où il se trouve, et non où la société dit qu'il est. Pierre rejette la longue-vue, selon la métaphore de Tolstoï, avec laquelle il voyait comme mesquin ce qui était autour de lui. Semblable en cela au récit que Levinas donne de sa captivité, Pierre, considérablement appauvri par la guerre, constate: «Ruiné, je suis devenu beaucoup plus riche». ${ }^{33}$ À la fin du roman, marié et père de famille, Pierre ne vit pas bourgeoisement en se contentant de faire prospérer au maximum

31 Tolstoï, 1972, p. 620-621.

32 Pierre ne se questionne plus sur le but de la vie car il a la foi. «ll ne pouvait avoir de but, parce qu'il possédait la foi, non pas une foi en des lois quelconques, ou des idées, mais la foi en un Dieu vivant, toujours senti personnellement. Autrefois, il le cherchait dans les buts qu'il s'assignait. Cette quête d'un but n'était que la quête de Dieu. En captivité, il avait compris subitement, non par des paroles et des raisonnements mais par un sentiment direct, ce que lui disait naguère sa nounou: "Dieu, Le voilà, ici, partout". En captivité, il avait compris que le Dieu de Karataïev était plus grand, plus infini, plus inconcevable que le Grand Architecte de l'univers que reconnaissaient les maçons. Il éprouvait le sentiment d'un homme qui aurait trouvé sous ses pieds ce qu'il cherchait, alors qu'il se fatiguait les yeux à regarder au loin. Toute sa vie, il avait regardé on ne sait où, par-dessus la tête des hommes qui l'entouraient; or il ne fallait pas se fatiguer les yeux, mais simplement regarder devant soi.» (lbid., p. 807).

33 Ibid., p. 814. Cette idée d'une captivité paradoxalement libératrice, comme chez Levinas, et positive se retrouve quand Pierre se demande ce qu'il répondrait si on lui demandait s'il voulait revenir «ce que [il] étai[t] avant [s]a captivité ou bien revivre de nouveau tout cela? Au nom du Ciel, encore une fois la captivité et la viande de cheval ! Nous pensons que dès qu'on nous sort des sentiers familiers, tout est perdu, c'est alors seulement que commence quelque chose de nouveau et de bon.» (Ibid., p. 831-832). 
ses affaires, mais se sent responsable du sort de sa communauté, et est animé d'un idéal.

Consonnant avec la description du bourgeois dans De l'évasion, le texte "Captivité», tiré du premier volume des Euvres de Levinas est le récit que Levinas fait de sa captivité en Allemagne pendant la seconde Guerre mondiale. Levinas y décrit le bourgeois comme l'être enfermé dans les apparences et les inauthentiques valeurs, clos sur lui-même et sur les biens et évoque ce que fut sa captivité dans la durée. Et à ce bourgeois, il oppose le prisonnier. ${ }^{34}$ Les prisonniers n'étaient pas bourgeois, car ils ont vécu une aventure authentique, contraire à l'idée de liberté illimitée, que connote parfois l'idée d'aventure, mais les prisonniers ont rencontré un véritable risque, un changement de vie réel et profond avec une mise en danger effective de leur existence. À cette mise en danger, à cet inconfort brutal de tout instant, Levinas oppose «le bourgeois [qui] est un homme installé. Il ne peut se soustraire au sérieux de la vie». ${ }^{35}$ Certes, le prisonnier, lui aussi est «installé» dans un camp, au sens de fixé, figé, rivé. Mais il ne l'est que corporellement, alors que le bourgeois est installé, au sens de rivé dans l'être. Il ne peut se soustraire à ce qu'il estime être sérieux de la vie, opérant un glissement de sens ; le sérieux, c'est, pour le bourgeois, les chiffres, c'est le quotidien, les affaires qu'on prend pour le cœur de l'existence; et là-dessus le bourgeois se trompe. Le bourgeois confond le sérieux authentique et l'esprit de sérieux, et c'est l'expérience de la captivité qui enseigne au prisonnier où se trouve le sérieux authentique de la vie, loin des affaires bourgeoises. Libre, le bourgeois est comme prisonnier de l'esprit de sérieux ; est, pour le bourgeois, sérieux ce qu'il considère tel, et qui ne l'est pas réellement. Pourquoi le prisonnier peut-il se soustraire à ce sérieux ? parce qu'il ne maîtrise rien et ne peut rien prévoir ou épargner, il doit s'adapter, il n'y a pas cadre à sa vie qui soit ferme et assuré d'être permanent.

34 Comme l'écrit Levinas, les prisonniers «n'ont pas été des bourgeois» (Levinas, 2009, p. 202). 
En outre, le bourgeois existe sans se soucier de son être propre, il est englué dans le quotidien, croyant que c'est de l'authentique, que le sens de la vie s'y trouve. Levinas poursuit sa description et énumère, en insistant sur les adjectifs possessifs, une somme de possessions bourgeoises: «Sa maison, son bureau, son cinéma, ses voisins, sont les points cardinaux de son existence». ${ }^{36}$ Le bourgeois s'oriente ainsi en fonction de ce qu'il possède. Aussi «sur le monde, sur le vaste monde [le bourgeois] n'ouvre que son journal et il l'ouvre comme une fenêtre. Il reste spectateur». ${ }^{37}$ Le bourgeois n'aperçoit du monde et de sa vie qu'une image, il ne vit sa vie que médiatement, il sait, mais ne vit pas, ne sort pas de lui-même ou de l'étendue de ses possessions, ne s'expose pas véritablement au dehors, à l'extérieur, à l'ailleurs. Ainsi les affaires du bourgeois, sa préoccupation essentielle, l'opposent-elles à la figure du prisonnier, telle que la dépeint Levinas dans "Captivité».

Le prisonnier, lui, par opposition, n'a pas tout ça, et en faisant l'expérience qu'il fait, il s'aperçoit que ce n'est important qu'en apparence, en surface. En effet, «le prisonnier, commente Levinas, comme un croyant, vivait dans l'au-delà». ${ }^{38}$ La réalité est pour lui ailleurs que dans le coin exigu du monde auquel il a accès, hors de son quotidien et il le savait. Contrairement au bourgeois, le prisonnier est libre car il a dû s'abstraire du quotidien qui se donne pour la réalité authentique mais n'est qu'une forme d'aliénation, d'affairement. La réalité perçue est décevante pour le prisonnier, qui sait qu'elle ne se limite pas à ce qu'il en voit, il croit en quelque chose de plus, de plus authentique et, pourrait-on dire, de plus réel que ce qui l'entoure immédiatement. Aussi, «il n'a jamais pris au sérieux le cadre étroit de sa vie», ${ }^{39}$ poursuit Levinas pour signifier qu'il n'accordait pas d'importance à son quotidien, à ce qu'il vivait présentement, mais se savait en périphérie de l'essentiel. On pourrait presque dire que les points cardinaux du prisonnier ne sont

36 Ibid.

37 Ibid.

38 Ibid.

39 lbid. 
pas les mêmes que ceux du bourgeois.

Décrivant le bourgeois comme prisonnier de son mode de vie et de pensée et lui opposant le prisonnier, sans liberté d'action, mais qui comprend, par sa réclusion et son enfermement même, où se trouve la liberté véritable, Levinas fait de l'expérience de la captivité une sorte de conversion, qu'ailleurs nous nommions un mouvement de désembourgeoisement. Il n'y a plus de propriété privée, plus de mien, pendant la captivité, ce qu'énonce ainsi Levinas:

Il y eut un dépouillement qui rendit le sens de l'essentiel. Pas toujours la pauvreté, pas toujours la faim, mais plus rien de strictement privé. Tous les espaces du quotidien devenus collectifs. Restait le lit: trois mètres cubes limités par les lits de vos deux voisins de gauche et de droite et de votre voisin du dessus. On possédait. Mais la propriété n'était pas votre maître [sous-entendu, elle l'est dans le cas du bourgeois et c'est peut-être elle qui est sérieuse], elle n'était plus sacrée. ${ }^{40}$

Et Levinas de conclure: «Nous avons appris la différence entre avoir et être. Nous avons appris le peu d'espace et le peu de choses qu'il faut pour vivre. Nous avons appris la liberté».41 Le texte se clôt sur un apparent paradoxe: apprendre la liberté en prison. "Nous avons appris», répété trois fois souligne la dimension formatrice et presque la conversion à laquelle conduit la captivité. La captivité apprend à ne plus être bourgeois, comme si l'humain moderne l'était constitutivement. Et Gérard Bensussan a raison de lire dans la paradoxale liberté du prisonnier un motif, que développera plus tard le Levinas de la maturité, selon lequel, je ne peux être pleinement et librement moi-même qu'en étant sorti de moi-même, désembourgeoisé et rendu responsable d'une responsabilité qui m'a toujours déjà incombé. ${ }^{42} \mathrm{D}$ 'ailleurs, Gérard Bensussan associe le bourgeois de ce texte au sujet de la philosophie occidentale qui se définit par un retour à soi et qu'il oppose à l'étranger tel

40 Ibid. On ne peut pas ne pas lire ici une attaque contre la sanctification de la propriété privée par la démocratie bourgeoise et la mise en évidence de son rôle dans la culture bourgeoise.

$41 \mathrm{Ibid} .$, p. 203.

42 Bernardo et Bensussan, 2013, p. 310. 
que le définit Levinas dans l'Humanisme de l'Autre Homme. ${ }^{43}$ Cette perception de la captivité qui libère et désembourgeoise, en faisant entrevoir ce qu'est l'existence authentique n'est pas sans rappeler, assez précisément, l'expérience, quasi initiatique, de la captivité de Pierre Bézoukhov, que nous avons analysée dans Guerre et paix, de Tolstoï.

Ainsi, si l'influence de la littérature russe sur la genèse de la pensée de Levinas est évidente pour de nombreux points, il nous semblé pertinent de mettre précisément au jour l'influence de Dostoïevski et de Tolstoï sur un point particulier de cette pensée: la figure du bourgeois. ${ }^{44}$ Cette figure possède chez Levinas - en particulier dans le portrait du bourgeois brossé au début de De l'évasion - des traits très similaires à ceux des personnages bourgeois de L'idiot de Dostoïevski. De façon plus originale, le bourgeois portraituré par Dostoïevski dans son «Essai sur le bourgeois» est doté des mêmes caractéristiques et surtout de la même philosophie que celle que Levinas comme «bourgeoise»: «la philosophie occidentale moderne». En effet, toutes deux font fond sur un sujet indépendant, égoïste, sourd aux souffrances des autres, semblable au «conatus» de Hobbes, avide d'avoir, de pouvoir et de valoir. À cette philosophie bourgeoise similaire, qu'ils condamnent

43 «Ce modèle de l'identité subjective, qu'on pourrait appeler génériquement philosophique (ontologique, phénoménologique, idéal), constitue de façon dominante toutes les représentations qui sont les nôtres de la subjectivité libre, d'une liberté qui se paie sans doute d'une perte mais pour gagner en authenticité et en vérité -c'est la figure "du retour à soi" ou encore du rassemblement en présent et représentation afin de se faire essence. Dans un texte des Carnets, intitulé "Captivité", cette figure de l'identité stable et assurée de soi est désignée de façon condensée, et littéraire, comme le "bourgeois" (...) On peut aisément rapporter ce "prisonnier", tel que Levinas le décrit très empiriquement et selon des déterminations historiques et politiques, à "l'étranger" tel qu'un passage d'Humanisme de l'Autre Homme en dégage les traits exemplaires. L'étranger désigne une ancienneté, un plus ancien que le modèle philosophique de l'identité subjective et autocentrée. II y a un plus-vieux que la conscience, un antérieur "à la conscience et au choix", un avant-l'identité subjective et libre. C'est "l'identité impossible", comme dit Levinas, d'une impossibilité plus vieille que l'identité c'est-à-dire d'une possibilité inactualisable dans une conscience ou une présence, d'une possible impossibilité que ni la métaphysique, ni la fin de la métaphysique ne peuvent nous faire entrevoir». (Bensussan, 2019).

44 Nous avons défendu la thèse que cette figure habite la pensée lévinassienne, même si elle est relativement discrète, dans notre thèse: «L'Exigence de désembourgeoisement: réflexion sur la figure du bourgeois dans la pensée d’Emmanuel Levinas et de Vladimir Jankélévitch» 
tous deux, Levinas et Dostoïevski opposent une philosophie autre, décentrée, soucieuse de l'altérité, dont le moteur principal ne serait ni la recherche du pouvoir ou du profit, mais le souci de l'autre, du commun et de la communauté dans la perspective dostoïevskienne, de la responsabilité éthique qui suspend le «conatus» pour Levinas. Enfin, le récit de sa captivité par Levinas présente une grande ressemblance avec celle de Pierre Bézoukof que peint Tolstoï, en particulier par le renversement qu'elles proposent toutes les deux - indépendamment de la réalité même de ces captivités - et qui fait du bourgeois soucieux de ses affaires un homme aveugle au sens véritable de la vie et plus prisonnier dans ses «affaires» que le prisonnier véritable qui comprend que le sens véritable de la vie n'est pas dans les affaires, mais dans une existence authentique purifiée et soucieuse des autres.

\section{Références}

ABENSOUR, Miguel. «Penser l'humain. Entre le métapolitique et le politique». Europe, no 991-992, Paris, oct.-nov. 2011, p. 2148.

BERNARDO, Fernanda et BENSUSSAN, Gérard. Os equívocos da ética: a propósito dos Carnets de Captivité de Levinas / Les équivoques de l'éthique: a propos des Carnets de Captivité de Levinas . Porto : Fundaçao Eng. António de Almeida, 2013.

BENSUSSAN, Gérard. «Humanismo, materialismo et politica em Levinas». Revista Ética e Filosofia Política, no XXII, Rio de Janeiro, 2019, pp. 35-56.

DOSTOÏEVSKI, Fédor. L'Idiot, traduit par Albert Mousset. Paris: Gallimard, 1994.

DOSTOÏEVSKI, Fédor. «Notes d'hiver sur des impressions d'été», traduit par Gustave Aucouturier: Récits, chroniques et polémiques. Paris: Gallimard, «Bibliothèque de la Pléiade», 1969.

LESCOURRET, Marie-Anne. Emmanuel Levinas. Paris: 
Flammarion, 1999, 1994.

LEVINAS, Emmanuel. De l'évasion. Paris: Le livre de poche, 1998a.

LEVINAS, Emmanuel. De Dieu qui vient à l'idée. Paris: Vrin, 1998b.

LEVINAS, Emmanuel. "Captivité». In: EEuvres 1: Carnets de captivité et autres inédits, publié sous la responsabilité de Rodolphe Calin et Catherine Chalier. Paris: Grasset/IMEC, 2009.

POIRIE, François. Emmanuel Lévinas. Qui êtes-vous? Lyon: La Manufacture, 1987.

SPARADRO, Rosario. «Levinas et la littérature russe». In: Levinas autrement, édité par R. Burggraeve, J. Hansel, M.-A. Lescourret, J.-F. Rey, J.-M. Salanski. Louvain-Paris: Éditions de l'institut supérieur de philosophie Louvain-la-neuve, 2012, pp. 43-58.

TOLSTOÏ, Léon. La Guerre et la Paix, traduction de Boris de Schloezer. Paris: Gallimard, 1972, deux volumes.

Recebido em: 01/02/2021

Aceito em: 22/03/2021

Publicado em abril de 2021 